\title{
Análise da qualidade temática em estudos envolvendo a pandemia Covid-19 à luz do design
}

\author{
Taynara de Sousa Mendes ${ }^{1}$; \\ Iraceles Cardoso Luzo ${ }^{2}$;
}

\section{resumo:}

Este estudo identifica a importância de análise da qualidade temática em estudos que contemplaram a pandemia Covid-19 e foram publicados, em eventos de design, no primeiro ano de pandemia no Brasil. Elenca conceitos teóricos práticos concernentes ao design e a pandemia Covid-19. Objetiva, de maneira geral, analisar as principais temáticas que abordem sobre a pandemia Covid-19, em estudos publicados em anais de eventos da área do design no ano de 2020, afim de identificar as principais discussões, contribuições, produtos e/ou ferramentas que estes trazem para a área e sociedade e, de maneira específica, a) apresentar os eventos de Design que serviram de base para coleta dos estudos que contemplaram a temática Covid-19; b) mapear os principais estudos dentro do Design abordando a pandemia Covid-19, publicados nos eventos da área no ano de 2020; c) identificar as principais temáticas presente nos estudos mapeados. Adota percurso metodológico composto por pesquisa bibliográfica pautada nas visões de teóricos concernentes as temáticas design, como Piscarreta (2013), Ponte e Niemeyer (2013), entre outros e sobre a pandemia Covid-19, como Santos (2020) e Freitas, Napimoga e Donalisio (2020) e coleta de dados realizada em anais publicados na Blucher Proceedings, no mês de maio de 2021, com universo total de 389 trabalhos distribuídos em 4 anais de eventos de design ocorridos e publicados entre os meses de outubro e dezembro de 2020. Constata que a temática precisa ser mais discutida e exposta, pois esses trabalhos são de crucial importância para um olhar crítico e científico sobre a temática, mais ainda por servirem de referências para a criação de outras pesquisas e refutações. Conclui que, entre os estudos analisados a maioria relatou problemas que surgiram diante do imediatismo causado pela pandemia, sendo possível identificar que o design foi utilizado para a busca de soluções, melhoria e adequação de espaços, serviços, ferramentas, tecnologias, em diversos tipos de iniciativas, o que demonstra seu caráter inter--plinar.

palavras-chave:

Covid-19; design; qualidade temática; eventos científicos.

\footnotetext{
${ }^{1}$ http://lattes.cnpq.br/4015514518427940

2 http://lattes.cnpq.br/5212899162840627
} 


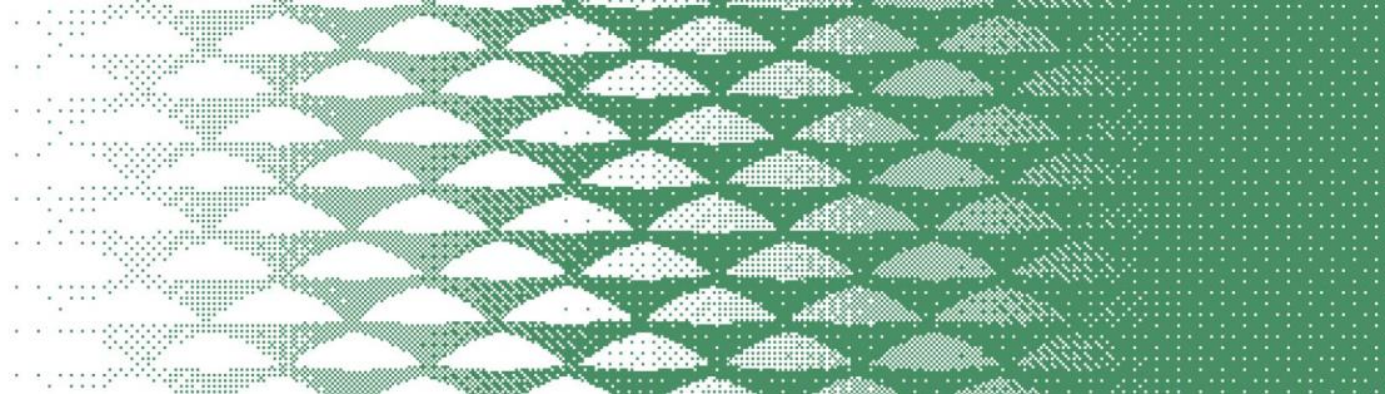

\section{Introdução}

O trabalho em Design é composto pela busca criativa para idealização e desenvolvimento de produtos, que atendam às necessidades de seus consumidores, independente do âmbito de produção - em série ou artesanal - dentro do nosso cotidiano, nos ambientes em geral. Os designers, profissionais atuantes em Design, planejam e criam objetos e/ou produtos através de uma ideia original, referências e/ou desejos de seus clientes, além de logomarcas, visual de páginas na internet, entre outros trabalhos, pensados e desenhados cuidadosamente.

No âmbito da produção científica, o Design se revela através de estudos que apontam novas tendências, sua interdisciplinaridade, suas contribuições para a sociedade - como a sustentabilidade e a acessibilidade, entre outros temas. Dessa maneira, mesmo com a crise sanitária e de saúde surgida com a pandemia Covid-19, pelo vírus SARS-CoV-2, que chegou ao Brasil em 2020, os eventos de Design tiveram continuidade, por meio de pesquisas e publicações, ainda que tenham sido parcialmente ou totalmente online, dadas as circunstancias da pandemia. Esses eventos consideraram importante a abordagem sobre a pandemia, dado que esse momento de crise afetou muitas pessoas, de maneira global, e, dessa forma, segue como um momento de adoção de novas estratégias de melhoria sociais e urbanas.

Diante desse cenário incerto e desafiador, este estudo visa responder o seguinte questionamento: Quais as principais discussões, produtos e/ou ferramentas abordando a pandemia Covid-19, incluindo suas contribuições, foram temas de estudos publicados nos principais eventos da área do Design no ano de 2020? Visando a elucidação dessa pergunta, o estudo propõe como objetivo geral, analisar as principais temáticas que abordem sobre a pandemia Covid-19, em estudos publicados em anais de eventos da área do Design no ano de 2020, afim de identificar as principais discussões, contribuições, produtos e/ou ferramentas que estes trazem para a área e sociedade. E como objetivos específicos: a) apresentar os eventos de Design que serviram de base para coleta dos estudos que contemplaram a temática Covid-19; b) mapear os principais estudos dentro do Design abordando a pandemia Covid-19, publicados nos eventos da área no ano de 2020; c) identificar as principais temáticas presente nos estudos mapeados.

Estudos dessa natureza se justificam no tocante às necessidades que surgiram e ainda podem surgir no contexto da pandemia e no pós-pandemia. Para tanto, esta pesquisa está dividida em seções, na seção 2 são abordados o contexto teórico prático em Design, revelando algumas de suas características, e a pandemia Covid-19, que demandou novos comportamentos dada sua emergência, na seção 3 é apresentado o percurso metodológico utilizado para a realização desta pesquisa, na seção 4 é demonstrada a análise da qualidade temática dos estudos envolvendo a pandemia Covid-19 à luz do Design e na seção 5 são apontadas considerações finais acerca da análise realizada, à guisa de uma conclusão, pois a pandemia segue em curso e ainda serão reveladas faces e emergências advindas desta crise mundial.

\section{Contexto teórico prático em design e a pandemia covid-19}

A criação e o desenvolvimento de produtos, por meio de planejamento, que levem em consideração a técnica e a estética, além da interação entre os usuários e esses produtos, remetem-se ao Design. Com o passar dos anos, os conceitos e terminologias aplicadas ao Design foram se modificando e a área apresentou interdisciplinaridade, no entanto, segue com o propósito de solução de problemas que surgem em diferentes ambientes na contemporaneidade, baseado na conquista de mercado e perspectiva de cenários futuros. Para Piscarreta (2013, p. 13) "O Design, num sentido mais global, é definido como a concepção e planeamento de todos os produtos feitos pelo homem e pode ser visto fundamentalmente como um instrumento para melhorar a qualidade de vida", desse modo, surge de um processo criativo, marcado pela industrialização, para atender as necessidades humanas.

No século XX, a indústria era marcada pela produção em série, conforme Ponte e Niemeyer (2013, p. 104) "A indústria se preocupava apenas em incrementar sua produção, sem se preocupar se, na realidade os objetos atendiam às necessidades e aos desejos dos consumidores", mas, depois de algum tempo isso se modificou, surgiu a indispensabilidade de contemplar a opinião do usuário e de reconhecer suas críticas, bem como atentar-se às mudanças provocadas pelas tecnologias de informação e 


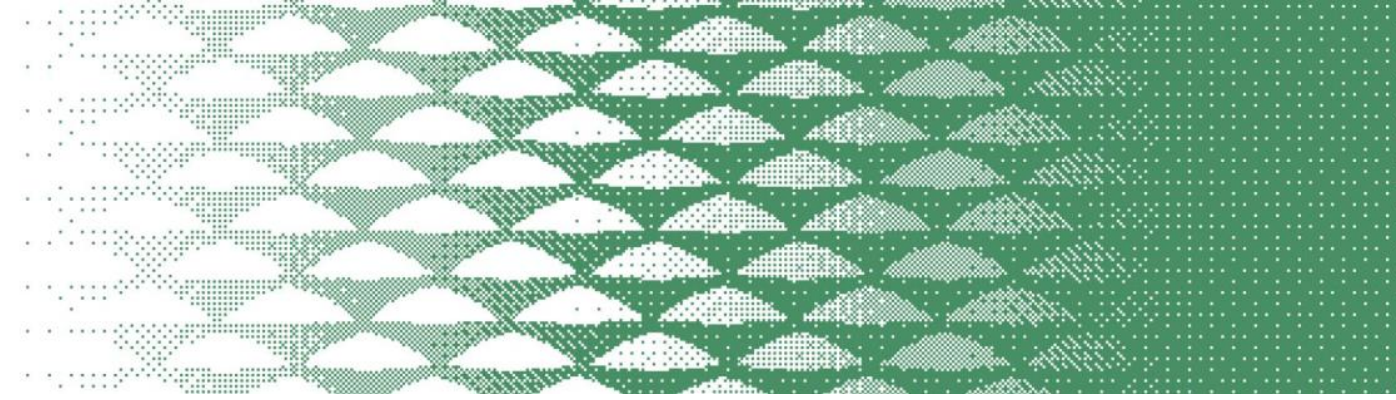

comunicação (TIC) e o interessante diálogo entre a arte a indústria, o bom resultado de um sistema (produto, ambiente ou mensagem) depende diretamente da sensação de bem-estar do usuário ao interagir com ele (BONI; SILVA; SILVA, 2014, p. 3).

Nesse cenário, o Design se faz útil na busca de alternativas de criação de projetos para ambientes e produtos acessíveis, sustentáveis e interativos, trabalhados em diferentes contextos pelos designers, como a web design, design da informação, design gráfico, design de interiores, design de moda, design de produtos industriais, design arquitetônico, design thinking, entre outros. Por conseguinte, o Design trabalha com a exteriorização intencional de ideias que são a resposta a um problema detectado (PONTE; NEIMEYER, 2013, p. 106), de onde nasce a inovação. Para Boni, Silva e Silva (2014, p. 2) "[...] devemos entender o Design como um ato mais racional que artístico devido sua dimensão e sua função em prol do benefício humano", ou seja, estabelecer conexão entre a ideia, o projeto, o resultado e o usuário final, sem separação, para o aumento de chances de sucesso de projetos idealizados.

Segundo Quintão e Triska (2014, p. 105), "Nos dias atuais, o design envolve a produção não só de objetos materiais, mas também de interfaces gráfico-digitais, com as quais o usuário interage no ciberespaço", pois algumas questões podem envolver representação, aspectos visuais, gráficos e linguagens inerentes a este espaço, dada a grande produção de informação e de conhecimento científico publicados e disponibilizados em sites, periódicos científicos, repositórios digitais, entre outros.

O Design, dada sua natureza, é desafiado e se expande em cenários de crise, a exemplo da pandemia Covid-19, pelo novo Coronavírus (SARS-CoV-2) que começou no fim de 2019, com suspeita de origem da China, a verdade é que, segundo a Organização Mundial de Saúde, a origem do vírus ainda não está determinada (SANTOS, 2020, p. 8), ele se propaga pelo ar - pelas gotículas de saliva, desencadeando sintomas gripais e altamente contagioso. O SARS-CoV-2 é um vírus respiratório diferente do vírus da influenza (gripe), cujo comportamento ainda não foi totalmente esclarecido (FREITAS; NAPIMOGA; DONALISIO, 2020, p. 4).

Mesmo com a rapidez da corrida de criação de vacinas, nem todas as pessoas foram imunizadas, no Brasil e em vários países do mundo o desafio da vacinação ainda repercute, além do surgimento de variantes do vírus, escassez de tratamento e medicamentos, falta de recursos, colapso na rede de saúde, crise política e corrupção. Não existiam planos estratégicos prontos para serem aplicados a uma pandemia de Coronavírus - tudo é novo (FREITAS; NAPIMOGA; DONALISIO, 2020, p. 1), foram e são necessárias a criação de estratégias para um novo "normal", ainda que após muitas mortes isso pareça impossível, pois durante o curso da pandemia desigualdades foram acentuadas, como a exclusão digital e a pobreza, houve acentuada precarização do trabalho informal, a multiplicação de notícias falsas, a economia seguiu entre altos e baixos e a crise sanitária é considerada alarmante e sem fronteiras.

A pandemia e a quarentena estão a revelar que são possíveis alternativas, que as sociedades se adaptam a novos modos de viver quando tal é necessário e sentido como correspondendo ao bem comum. Esta situação torna-se propícia a que se pense em alternativas ao modo de viver, de produzir, de consumir e de conviver nestes primeiros anos do século XXI (SANTOS, 2020, p. 29).

Uma das formas de combater a contaminação pelo vírus é o distanciamento social, que naturalmente alterou as rotinas em diversos ambientes, empresas, comércio, instituições de ensino, entre outros, já que alguns locais puderam optar pelo teletrabalho (home-office), ou então pela quarentena e/ou afastamento, a tragédia é que neste caso a melhor maneira de sermos solidários uns com os outros é isolarmo-nos uns dos outros e nem sequer nos tocarmos (SANTOS, 2020, p. 7), o distanciamento social é uma das recomendações da Organização Mundial da Saúde (OMS) para o combate ao Coronavírus.

Isso foi possível com o aumento considerável do uso da internet e de dispositivos digitais, para que as atividades não parassem totalmente. Assim, seguiram-se o curso, desde 2020, de alguns eventos científicos de Universidades e instituições de ensino que se preocuparam em discutir os principais impactos causados por esta pandemia, a fim de desvendar novos caminhos para esse momento e para um cenário de pós-pandemia. 


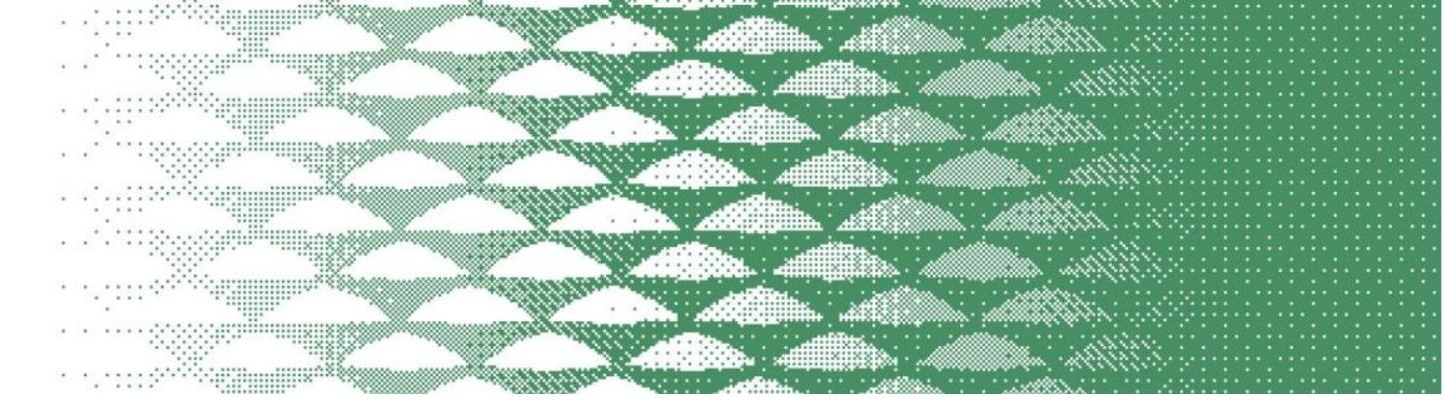

\section{Percurso metodológico}

Este estudo, em relação aos seus objetivos caracteriza-se como descritivo, quando busca analisar e interpretar os dados obtidos por meio de análise da qualidade temática dos estudos publicados nos anais de eventos da área do design, sem a necessidade de manipulá-los. Andrade (2003, p. 124), afirma que na pesquisa descritiva "[...] os fatos são observados, registrados, analisados, classificados e interpretados, sem que o pesquisador interfira neles". Foi realizada, inicialmente, uma pesquisa bibliográfica que consiste na "[...] investigação das produções bibliográficas relacionadas ao assunto estudado e o posterior recolhimento dessas fontes de informação" (PRODANOV; FREITAS, 2013, p. 75), a fim de conhecer e aproximar-se dos temas mencionados neste estudo.

Classifica-se ainda como uma pesquisa de abordagem qualitativa, uma vez que busca descrever determinado fenômeno a partir da interação com os objetivos estabelecidos na investigação, levantando aspectos significativos em torno do objeto estudado (SILVEIRA; CÓRDOVA, 2009). Nesse aspecto, a descrição vai além do comum quando interpreta as ações e entende suas particularidades.

A coleta de dados foi realizada em anais publicados na Blucher Proceedings, no mês de maio de 2021. Para a escolha da amostra de pesquisa, teve-se como universo um total de 389 trabalhos distribuídos em 4 anais de eventos de design ocorridos e publicados entre os meses de outubro e dezembro de 2020, sendo estes: Colóquio Internacional de Design 2020 (CID 2020), XXIV Congresso Internacional da Sociedade Ibero-americana de Gráfica Digital (SIGRADI 2020), VIII Encontro Nacional de Ergonomia do Ambiente Construído e IX Seminário Brasileiro de Acessibilidade Integral (ENEAC 2020) e o $3^{\circ}$ Seminário de Pesquisa do Programa de Pós-Graduação em Design da FAUUSP (III SPG DSG FAUUSP).

\begin{tabular}{|c|c|c|c|}
\hline \multicolumn{1}{|c|}{ Quadro 1 - Universo, tipologia e amostra da pesquisa } \\
\hline EVENTO & $\begin{array}{c}\text { Total de trabalhos } \\
\text { publicados }\end{array}$ & Tipo de trabalhos & $\begin{array}{c}\text { Trabalhos com } \\
\text { a temática } \\
\text { estudada }\end{array}$ \\
\hline CID 2020 & 120 & Artigos completos & 11 \\
\hline SIGRADI 2020 & 135 & Artigos completos & 5 \\
\hline ENEAC 2020 & 101 & $\begin{array}{c}\text { Resumos, Resumos } \\
\text { expandidos e Artigos } \\
\text { completos }\end{array}$ & 0 \\
\hline III SPG DSG FAUUSP & 33 & Resumos & 0 \\
\hline
\end{tabular}

Fonte: Adaptado de Blucher Proceedings (2021).

Levando em consideração o objetivo da pesquisa, apenas estudos que abordassem a temática foram escolhidos. Logo, a amostra final é composta por 16 trabalhos, que foram recuperados por palavras-chave, como: pandemia e covid-19.

\section{Análise da qualidade temática dos estudos envolvendo a pandemia covid-19 à luz do design}

Cumprindo os objetivos operacionais estabelecidos, nesta seção segue-se para a identificação e análise da qualidade temática dos estudos mapeados (Apêndice A).

Dos 389 trabalhos apresentados e publicados nos anais dos eventos analisados, apenas 16 abordavam a pandemia Covid-19 como tema de suas pesquisas. Esse número equivale a $4,11 \%$ do total, o que revela que a temática precisa ser mais discutida e exposta, pois esses trabalhos são de crucial importância para um olhar crítico e científico sobre a temática, mais ainda por servirem de referências para a criação de outras pesquisas e refutações.

Para análise das temáticas, dividimos os trabalhos em 6 categorias, sendo elas: 1 - O design e a busca de soluções para o ambiente de trabalho ou assunto correlato; 2 - $\mathrm{O}$ design na criação de protótipos; 3 - O design em iniciativas para a produção de itens para o combate ao vírus; 4 - $\mathrm{O}$ design 


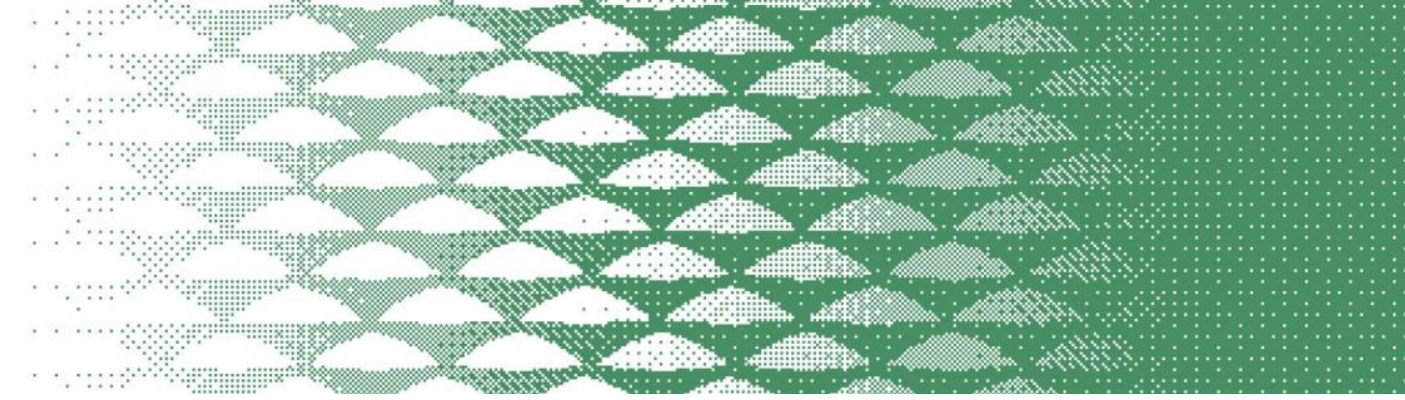

como método para transformação e/ou adequação de algum serviço com a nova realidade; 5 - $\mathrm{O}$ design e a emoção humana; 6 - $\mathrm{O}$ design estratégico e a construção arquitetônica.

Referente a primeira categoria, 3 estudos abordaram questões relacionadas a emprego, trabalho e suas condições. Enquanto um estudo mostrou como uma empresa remodelou todo seu serviço, adequando-o e levando-o para o espaço digital, fazendo uso de soluções tecnológicas, como o uso de softwares 3D no processo de design, os outros dois focaram no trabalhador e suas dificuldades no período pandêmico. O primeiro, trabalhou a ergonomia como ferramenta para a melhoria da qualidade de vida dos trabalhadores que foram o home office, sendo esta (ergonomia) vista além da sua forma técnica, mas também do viés ético, pensando no bem-estar das pessoas que foram obrigadas, mediante a emergência da situação, a uma transformação radical sem aviso prévio. $O$ segundo, relatou a criação de um protótipo de aplicativo que visava conectar jovens desempregados a oferecer seus serviços, de acordo com a sua formação, mediante as necessidades e demandas que surgissem em suas comunidades. Tal iniciativa, é resultado de trabalho cooperativo entre designers e pesquisadores, por meio da ferramenta metodológica do Human Centered Design, e visava ajudar principalmente os jovens a enfrentar a realidade do cenário atual, onde de acordo reportagem de Campos (2021) do site Agência Brasil:

Os trabalhadores com idade entre 18 e 24 anos foram os mais prejudicados pela pandemia [...] A taxa de desocupação subiu de $23,8 \%$ no quarto trimestre de 2019 para $29,8 \%$ no mesmo período de 2020 , o que corresponde a quase 4,1 milhões de jovens à procura de emprego. (CAMPOS, 2021).

Esse mesmo estudo faz parte, também, da segunda categoria, O design na criação de protótipos, que conta ainda com a pesquisa que relata o processo de prototipagem de um modelo de redes, que visava analisar as relações entre os diferentes atores no desenvolvimento da vacina para a covid 19. Para tal, os pesquisadores fizeram uso do design estratégico, através da abordagem de redes, para a criação do protótipo simples, mediante a situação emergente causada pela pandemia. Flores, Borelli e Costa (2020, p. 580): “[...] a interpretação das redes permite identificar de maneira visual e precisa os atores e suas relações, pouco interferindo no resultado final. Por meio das redes, é possível também identificar os papeis atribuídos aos atores, como uma generalização das suas funções no contexto integrado.

$\mathrm{Na}$ terceira categoria, os estudos (03) focam em iniciativas colaborativas entre designers, pesquisadores, alunos de design de universidades públicas e institutos, e nos seus esforços na elaboração, produção e distribuição dos mais diversos itens que pudessem ser usados para a proteção e combate ao coronavírus. Entre os itens mais citados nos estudos estão: máscaras e aventais de tecidos, face Shields, capuz de proteção de orelhas e pescoço para profissionais da linha de frente, máscaras cirúrgicas, além de materiais que contribuíam também no combate a desinformação da população, com a produção e distribuição de materiais de comunicação com informações claras sobre prevenção ao vírus. O que todas essas iniciativas apresentadas nos estudos mostram, correspondem ao quanto:

Universidades públicas e institutos federais são indispensáveis para vencermos essa batalha e atenuarmos suas consequências. O designer deve continuar olhando para os diferentes contextos, ter empatia e responsabilidade social, utilizar capacidades e habilidades de design para contribuir ainda mais com a sociedade nestes tempos. (OLIVEIRA, 2020, p. 1474).

Em relação a quarta categoria, dois estudos abordaram as transformações que foram exigidas dado ao cenário emergente causado pela pandemia. Um dos estudos mostrou como três empresas de moda lidaram com o período pandêmico no início de 2020, sendo as principais transformações e adaptações ligadas a intensificação do uso de ferramentas ligadas à internet e a tecnologia, para lidarem com todas necessidades do setor, que iriam desde logística, desfiles de forma online, a comunicação final com o cliente. $\mathrm{O}$ outro estudo trata da mudança relacionada a grupo especifico de manifestação 


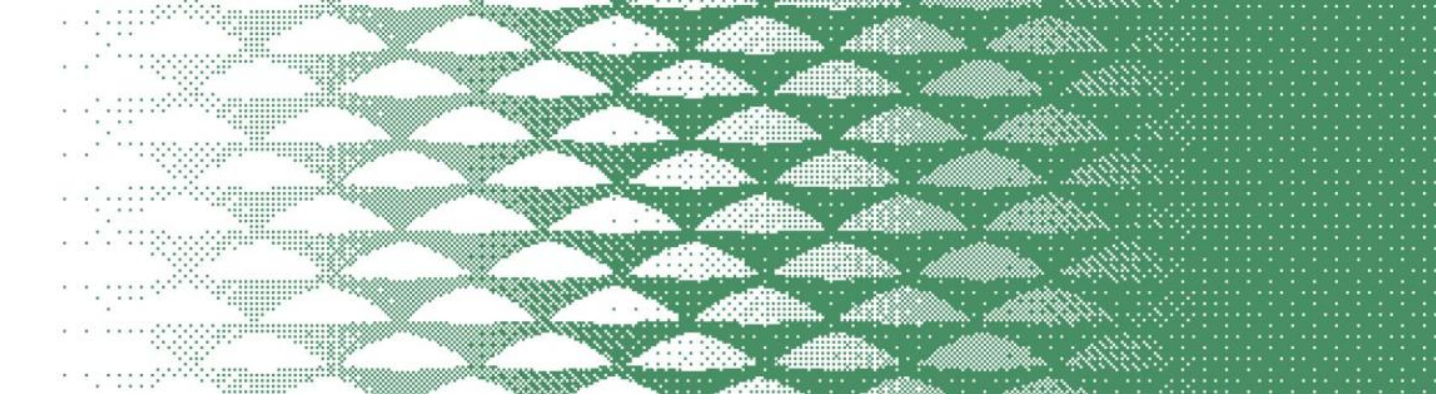

metodológica do HCD (Design Centrado no Humano), a aceleração da transformação digital na Moda Virtual revelando novos fluxos de trabalho e oportunidades resultantes do uso de softwares 3D no processo de Design. Além do movimento Maker com uma perspectiva coletivista e inclusiva que aborda produções associadas ao ideário do movimento "faça você mesmo", as ações das Universidades e Institutos federais, especialmente dos cursos e departamentos de Design como produção de máscaras de tecido (caseira) para a sociedade, produção de EPIs para os profissionais da saúde, produção de materiais gráficos informacionais para prevenção da COVID-19, entre outras produções, a Ergonomia como alternativa para qualidade de vida para estimular percepções e reflexões sobre a implementação de uma cultura organizacional de bem-estar no trabalho, as transformações arquitetônicas, visando sustentabilidade e construção de edifícios inteligentes com baixo custo e realizados por meio de projetos colaborativos, entre outros. Tais criações e abordagens revelaram a importância desses estudos de Design em meio a situação complexa que vivemos, por meio de inovação, estratégias e adaptação a essa realidade mundial.

Quanto aos objetivos específicos, estes foram atendidos, pois foi possível apresentar quais eventos em Design contemplaram a Covid-19 em seus trabalhos no ano de 2020, sendo eles o Colóquio Internacional de Design e o XXIV Congresso Internacional da Sociedade Ibero-americana de Gráfica Digital, nesses eventos foram mapeados 16 trabalhos que abordaram a pandemia Covid-19 e a identificação de suas temáticas que variaram entre Ergonomia, Moda, Educação, Visualização de dados, prototipagem de modelos de redes, transformações arquitetônicas e outros.

Como contribuição, foi possível identificar que o Design foi utilizado para a busca de soluções, melhoria e adequação de espaços, serviços, ferramentas, tecnologias, em diversos tipos de iniciativas, o que demonstra seu caráter interdisciplinar. Como o cenário pandêmico ainda se encontra assolado em boa parte do mundo, e mesmo com a vacinação já em andamento pelo globo, para trabalhos futuros se faz necessário que mais estudos envolvendo o design e suas competências, iniciativas, projetos, e desenvolvimento de produtos sejam feitos, ou seja, mais pesquisas e estudos precisam ser realizados, com foco na busca de solução para problemas complexos, melhoria dos serviços e consequentemente, criação de estratégias para tentar diminuir as desigualdades acentuadas na pandemia e que irão se revelar no pós-pandemia.

\section{Thematic quality analysis in studies involving the covid-19 pandemic in the light of design}

\section{abstract:}

This study identifies the importance of thematic quality analysis in studies that covered the Covid-19 pandemic and were published, in design events, in the first year of the pandemic in Brazil. It lists practical theoretical concepts concerning Covid-19's design and pandemic. It aims, in general, to analyze the main themes that address the Covid-19 pandemic, in studies published in the annals of design events in the year 2020, in order to identify the main discussions, contributions, products and / or tools that they bring to the area and society and, in a specific way, a) to present the design events that served as a basis for collecting the studies that contemplated the Covid-19 theme; b) map the main studies within design addressing the Covid-19 pandemic, published in events in the area in the year 2020; c) to identify the main themes present in the mapped studies. It adopts a methodological path composed of bibliographic research based on the views of theorists concerning design themes, such as Piscarreta (2013), Ponte and Niemeyer (2013), among others, and on the Covid-19 pandemic, such as Santos (2020) and Freitas, Napimoga and Donalisio (2020) and data collection carried out in annals published in Blucher Proceedings, in the month of May 2021, with a total universe of 389 works distributed in 4 annals of design events that occurred and published between the months of october and december 2020. Notes that the theme needs to be further discussed and exposed, as these works are of crucial 


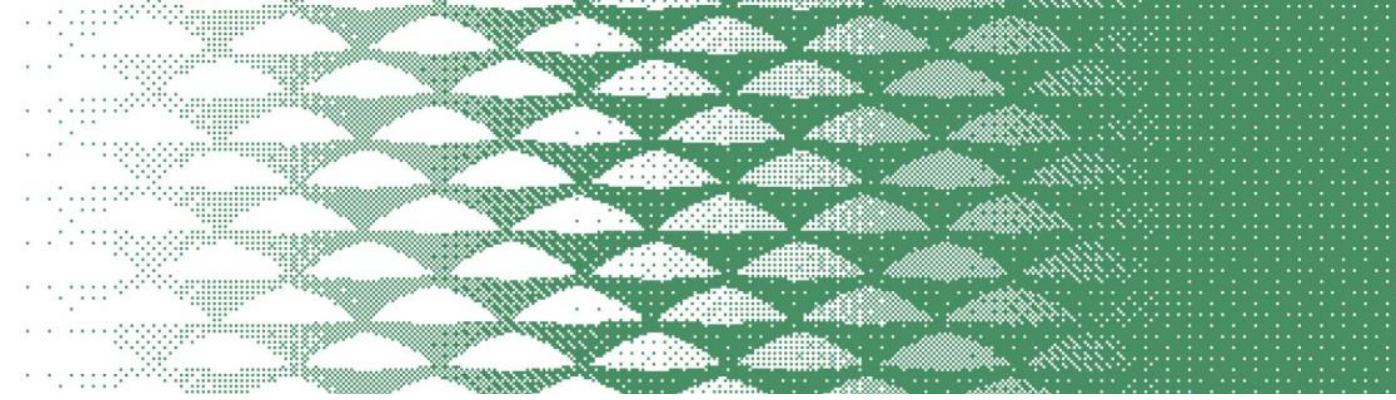

importance for a critical and scientific look at the theme, even more because they serve as references for the creation of other research and refutations. It concludes that, among the studies analyzed, most reported problems that arose due to the immediacy caused by the pandemic, making it possible to identify that the design was used to search for solutions, improvement and adequacy of spaces, services, tools, technologies, in several types of initiatives, which demonstrates its interdisciplinary character.

keywords:

Covid-19; design; quality analysis; scientific events.

\section{Referências bibliográficas}

ANDRADE, Maria Margarida de. Introdução à metodologia do trabalho científico. São Paulo: Atlas, 2003. Cap. 10-11.

BONI, Claudio Roberto; SILVA, Kelenson; SILVA, José Carlos Plácido da. A (IN)DEFINIÇÃO DO DESIGN: os limites do design e a relação com a arte e a indústria. In: CONGRESSO BRASILEIRO DE PESQUISA E DESENVOLVIMENTO EM DESIGN, 11., 2014, Gramado. Anais [...]. Gramado: UFRS, 2014. P. 1-11. Disponível em: https://www.proceedings.blucher.com.br/article-details/aindefinio-do-design-os-limites-do-design-e-a-relao-com-a-arte-e-a-indstria-12656. Acesso em: 30 abr. 2021.

CAMPOS, Ana Cristina. Agência Brasil. Impacto da pandemia é maior para trabalhadores jovens, diz Ipea. 2021. Disponível em: https://agenciabrasil.ebc.com.br/economia/noticia/2021-04/impactoda-pandemia-e-maior-para-trabalhadores-jovens-diz-ipea. Acesso em: 28 maio 2021.

FLORES, Guilherme Pedó; BORELLI, Wyllians Vendramini; COSTA, Filipe Campelo Xavier da. Prototipagem de modelo de redes para identificar relações principais no desenvolvimento da vacina para a COVID-19. In: COLÓQUIO INTERNACIONAL DE DESIGN, 2020, Edição Virtual. Anais [...]. Minas Gerais: UEMG, 2020. P. 576-586. Disponível em: http://pdf.blucher.com.br.s3-sa-east1.amazonaws.com/designproceedings/cid2020/110.pdf. Acesso em: 28 maio 2021.

FREITAS, André Ricardo Ribas; NAPIMOGA, Marcelo; DONALISIO, Maria Rita. Análise da gravidade da pandemia de Covid-19. Epidemiologia e Serviços de Saúde, [S.L.], v. 29, n. 2, p. 1-5, abr. 2020. FapUNIFESP (SciELO). Disponível em: https://www.scielosp.org/article/ress/2020.v29n2/e2020119/. Acesso em: 29 abr. 2021.

MURTA, Gabriella; MONT'ALVÃO, Claudia; KOSMINSKY, Doris. Visualização de Dados - Uma análise do redesign de visualizações da COVID-19. In: COLÓQUIO INTERNACIONAL DE DESIGN, 2020, Edição Virtual. Anais [...]. Minas Gerais: UEMG, 2020. P. 1600-1614. Disponível em: http://pdf.blucher.com.br.s3-sa-east-1.amazonaws.com/designproceedings/cid2020/120.pdf. Acesso em: 29 maio 2021.

OLIVEIRA, Lorena Gomes Ribeiro de. Design em tempos de pandemia: contribuições de universidades públicas e institutos federais. In: COLÓQUIO INTERNACIONAL DE DESIGN, 2020, Edição Virtual. Anais [...]. Minas Gerais: UEMG, 2020. P. 1468-1476. Disponível em: http://pdf.blucher.com.br.s3-sa-east-1.amazonaws.com/designproceedings/cid2020/45.pdf. Acesso em: 28 maio 2021.

QUINTÃO, Fernanda S; TRISKA, Ricardo. Design de informação em interfaces digitais: origens, definições e fundamentos. Revista Brasileira de Design da Informação, São Paulo, v. 11, n. 1, p. 106-118, 2014. Disponível em: https://www.infodesign.org.br/infodesign/article/view/243. Acesso em: 01 maio 2021.

PISCARRETA, Inês da Costa Pina de Sousa. TEORIA E PRÁTICA NO DESIGN. 2013. 50 f. Dissertação (Mestrado em Produção, Edição e Comunicação de Conteúdos) - Universidade do 


\begin{tabular}{|c|c|}
\hline 11 & $\begin{array}{l}\text { A Ergonomia como alternativa teórico metodológica em prol da Qualidade de Vida } \\
\text { no Trabalho no contexto Pandêmico da COVID-19 }\end{array}$ \\
\hline \multicolumn{2}{|r|}{$\begin{array}{c}\text { EVENTO: XXIV Congresso Internacional da Sociedade Ibero-americana de Gráfica } \\
\text { Digital }\end{array}$} \\
\hline $\mathbf{N}^{\mathbf{0}}$ & \\
\hline 1 & $\begin{array}{l}\text { Transformações arquitetônicas no cc } \\
\text { a uma construção resiliente, sustent }\end{array}$ \\
\hline 2 & $\begin{array}{l}\text { A imp } \\
\text { do CO }\end{array}$ \\
\hline 3 & rução após a emergência do \\
\hline 4 & odelagem Spatiotemporal de COVID-19 Propagaçâ \\
\hline 5 & \\
\hline
\end{tabular}

Fonte: Adaptado de Blucher Proceedings (2021). 